\title{
Nishikawa Prize article: Electron lenses, Tevatron, and selected topics in accelerators ${ }^{1}$
}

\author{
Vladimir Shiltsev \\ Fermi National Accelerator Laboratory, P.O. Box 500, Batavia, Illinois 60510, USA
}

(Received 27 May 2019; published 24 July 2019)

\begin{abstract}
This article is an extended version of the talk is given at the IPAC19 (Melbourne, Australia, May 2019) on the occasion of acceptance of the ACFA/IPAC19 Nishikawa Tetsuji Prize for a recent, significant, original contribution to the accelerator field, with no age limit with citation "...for original work on electron lenses in synchrotron colliders, outstanding contribution to the construction and operation of high-energy, high-luminosity hadron colliders and for tireless leadership in the accelerator community."
\end{abstract}

DOI: 10.1103/PhysRevAccelBeams.22.074801

\section{TRIBUTE TO NISHIKAWA TETSUJI}

It is a great honor for me to receive the 2019 ACFA/IPAC Nishikawa Tetsuji Prize. Professor Nishikawa-see Fig. 1was among the pioneers of particle accelerators who shaped our field at its early stage. After making a significant contribution to the Brookhaven National Laboratory (BNL) linac in 1964-1966, he moved to Japan and established Japan National Lab for High Energy Physics in 1969 (now KEK). There he initiated design, construction, commissioning, and operation of such remarkable facilities as the $12 \mathrm{GeV}$ proton synchrotron [1], neutron facility J-PARC, the $500 \mathrm{MeV}$ cancer treatment synchrotron, the KEK Photon Factory, and TRISTAN electron-positron collider. Professor Nishikawa was also a man of great integrity and an active supporter of the international cooperation, in particular, between Japan and the United States [2].

In this article, I summarize the activities of myself and my teams noted in the prize citation and briefly discuss the topics of my current research.

\section{ELECTRON LENSES}

I got a Ph.D. in accelerator and beam physics from BINP (Novosibirsk, Russia) in 1994 and worked at the leading accelerator laboratories in Protvino (Russia), the SSCL (USA), and DESY (Germany) before joining Fermilab in 1996, where shortly thereafter I initiated the project of beam-beam compensation with Tevatron electron lenses (TELs) [3-5]. Since then, the electron lenses-a novel

\footnotetext{
${ }^{1}$ The 2019 IPAC Tetsuji Nishikawa prize was won by Vladimir Shiltsev. This paper is an invited contribution inspired by this award.

Published by the American Physical Society under the terms of the Creative Commons Attribution 4.0 International license. Further distribution of this work must maintain attribution to the author(s) and the published article's title, journal citation, and DOI.
}

instrument for high-energy particle accelerators-have been added to the toolbox of modern beam facilities, being particularly useful for the energy frontier superconducting hadron colliders ("supercolliders") [6]. The physics mechanism of the electron lens is the space-force of a narrow and long beam of low-energy electrons, immersed in a strong longitudinal magnetic field. The Lorentz force of a lowenergy electron beam $\beta_{e} c$ with velocity and current density distribution $j_{e}(r)$ is

$$
e\left(E_{r}+B_{\theta}\right)=\frac{4 \pi e\left(1+\beta_{e}\right)}{\beta_{e} c} \frac{1}{r} \int_{0}^{r} j_{e}\left(r^{\prime}\right) r^{\prime} d r^{\prime} .
$$

It effectively acts on the accelerator's high-energy beam moving along (or colliding) with the electron beam, while the effect of the longitudinal magnetic field is usually a minor or easily correctable imperfection. The transverse motion of electrons is essentially frozen along the magnetic field lines which, therefore, assures outstanding stability of the electron beam. Given that the electron beam transverse shapes and longitudinal current modulation patterns can be broadly varied (usually, created at an electron gun) [7], the electron lenses have become a uniquely flexible instrument.

The electron lenses for supercolliders were originally considered in 1993 by Tsyganov, Taratin, and Zinchenko [8] to reduce the tune spread due to beam-beam interactions in the Superconducting Super Collider (SSC), and, independently, in 1997 Shiltsev et al. proposed them for compensation of the long-range and head-on beam-beam effects in the Tevatron proton-antiproton collider [3,4]. A comprehensive theory of the electron lens beam-beam compensation and detail design considerations were accomplished by 1999 [5]. Then, at Fermilab, the first two TELs were designed, built, and installed in the Tevatron $1.96 \mathrm{TeV}$ c.m.e. proton-antiproton collider in 2001 and 2004 (see Fig. 2), operated till the end of run II in 2001, and were used for compensation of long-range beam-beam effects (the TELs varied the tune shift of selected individual $0.98 \mathrm{TeV}$ 


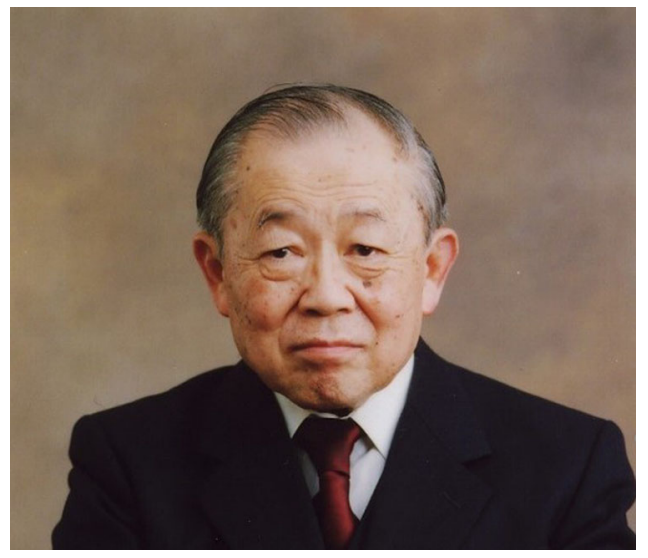

FIG. 1. Tetsuji Nishikawa (1926-2010).

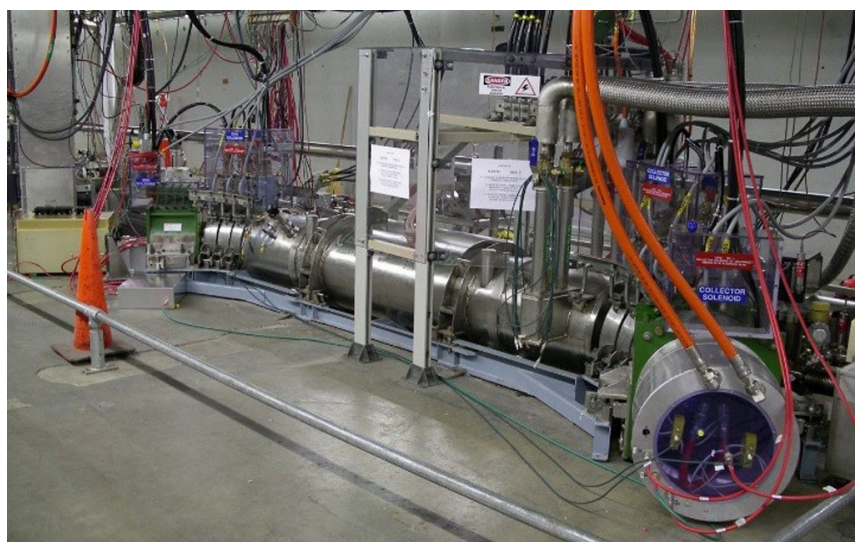

FIG. 2. Tevatron electron lens in the accelerator tunnel.

antiproton or proton bunches by $0.003-0.01$ ) [9]—-see Fig. 3; longitudinal collimation-removal of the dc beam particles from the abort gaps-for $10 \mathrm{yr}$ in regular operation [10]; studies of head-on beam-beam compensation [11,12]; and

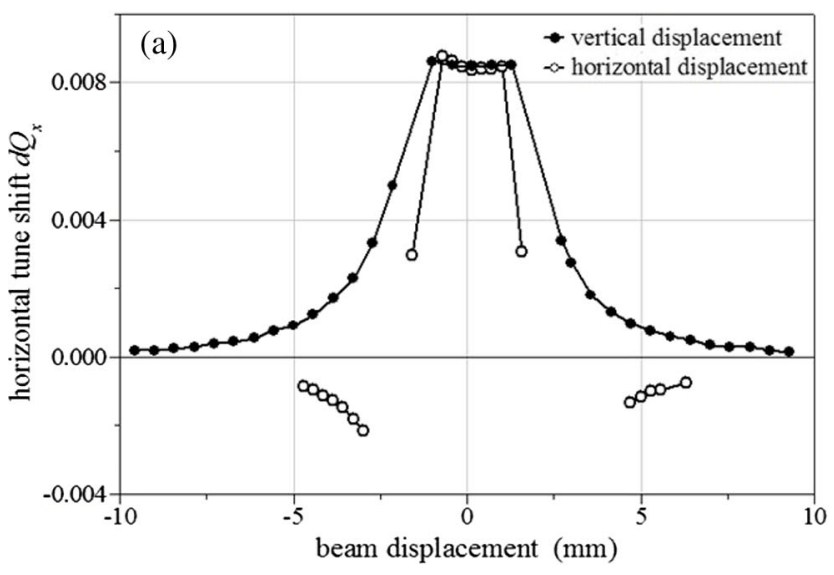

demonstration of halo scraping with hollow electron beams $[13,14]$. Since 2015, two electron lenses are installed in the Relativistic Heavy Ion Collider at BNL and very successfully used for head-on beam-beam compensation leading to doubling the luminosity in proton-proton collisions [15].

Present worldwide efforts on the electron lenses cover several areas of research: (a) hollow electron beam collimation of protons in the HL-LHC [16,17]; (b) long-range beambeam compensation with electron lenses as current-bearing "wires" in the HL-LHC [18-20]; (c) generation of nonlinear integrable lattices with special transverse current distribution $j_{e}(r) \sim /\left(1+r^{2}\right)^{2}$-first proposed in Refs. [5,21]-e.g., in the Integrable Optics Test Accelerator (IOTA) ring [22,23]; (d) Landau damping of a broad spectrum of coherent instabilities by the electron lens-induced tune spread $[13,24]$ in, e.g., the LHC, FCC-hh (where an electron lens can outperform some 10000 octupoles), or FNAL Recycler Ring; (e) compensation of space-charge effects in modern high-intensity rapid cycling synchrotrons (RCS) [25,26].

The electron lenses usually employ a low-energy ( $10 \mathrm{kV})$, high current (1-10 A) millimeter or submillimeter size magnetized electron beam to affect beneficially a high-energy beam of hadrons (protons, antiprotons). The design of the lenses required advancing several technologies: high field quality solenoids and correctors (sometimes superconducting, with up to a 4-6 T field), high-brightness electron beam generation and low-loss transport (gun, collector, etc.), fast high-voltage (HV) gun anode modulator (10 kV, hundreds of microseconds, and multiples of the machine revolution frequency, e.g., $50 \mathrm{kHz}$ in the Tevatron), sophisticated power recirculation electrical scheme, ultrahigh vacuum, and beam diagnostics system.

Together with other beam physics R\&D, further research on the electron lenses motivated the construction of a dedicated accelerator test facility-IOTA ring at

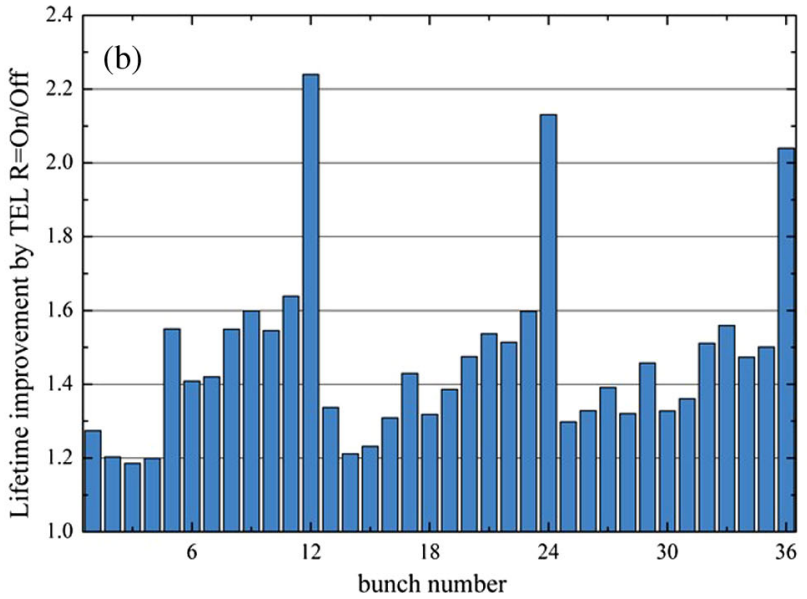

FIG. 3. (a) Left: TEL1-induced shift of a $980 \mathrm{GeV}$ proton horizontal betatron tune versus vertical (filled circles) and horizontal (open circles) electron beam displacement $\left(J_{e}=1 \mathrm{~A}, U_{c}=6.0 \mathrm{kV}\right.$, electron gun with uniform transverse current distribution); (b) TEL-2 in the dc regime significantly improves the lifetime of all 36 proton bunches early in the Tevatron store \#5183 with initial luminosity $L=253 \times 10^{30} \mathrm{~cm}^{-2} \mathrm{~s}^{-1}$ - from Ref. [11]. 


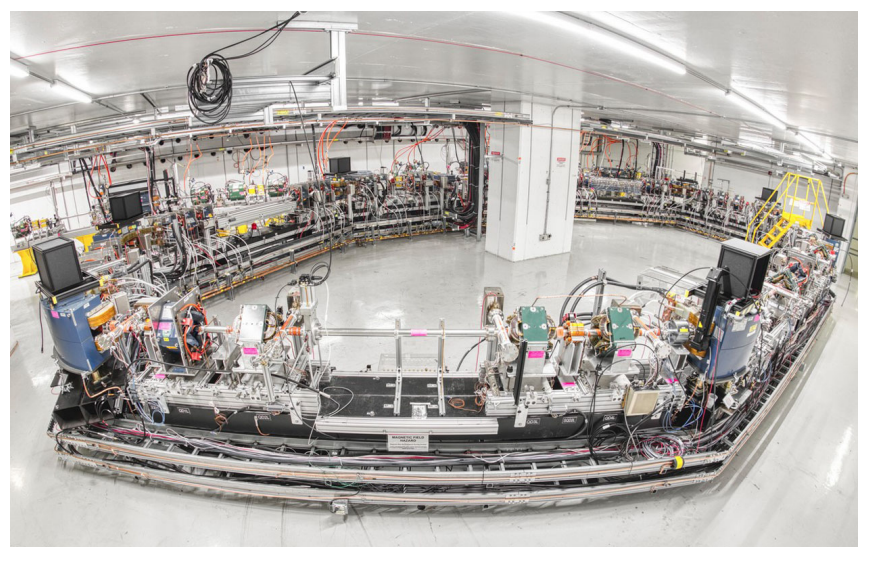

FIG. 4. Fermilab's IOTA ring for beam physics research.

Fermilab [27]. The 40-m circumference ring, capable of operating with $70-150 \mathrm{MeV} / \mathrm{c}$ electrons and protonssee Fig. 4-has been commissioned in 2018 and the first experimental run with electrons has taken place in October, 2018-March, 2019, providing many excellent results in key experiments on the nonlinear integrable optics, single-electron tomography, and many otherssee, e.g., related reports at the IPAC'19 [28-45]. The experiment on space-charge compensation with an electron lens is being prepared with the goal to be ready for the IOTA operation with protons in 2020-2021.

\section{TEVATRON COLLIDER IMPROVEMENTS}

Over more than three decades in accelerators, I have been involved in, contributed to, or led the design, construction, or operation of a number of forefront accelerators and colliders, such as Tevatron, VLEPP, TESLA, UNK, HERA, PIP-II, LHC, Muon Collider, ILC, etc. The most extensive work was done for the Tevatron collider which took over a decade [46-49]. Through computer modeling and a series of

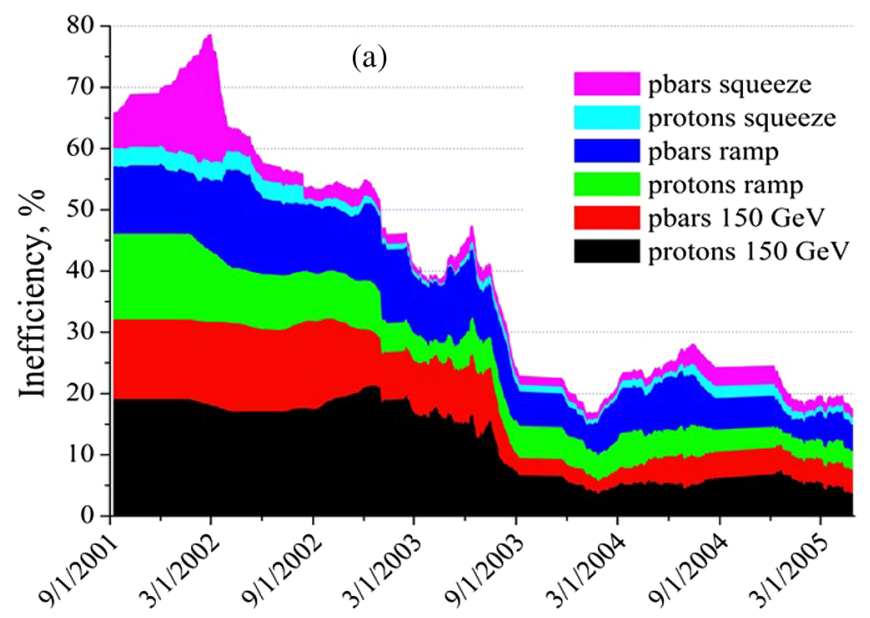

dedicated beam studies we greatly advanced the understanding of a number of critical beam physics phenomena creating impediments on the collider's luminosity, such as long-range and head-on beam-beam effects [49], beam injection optics matching, control of coherent beam instabilities, two-stage halo collimation, novel collimation methods by the hollow electron beams collimation method [12], and bent crystals [50]. The beam-beam effects significantly affected the collider operation and resulted in either emittance growth or bunch length reduction or particle losses at all stages from injection to energy ramp and squeeze as well as in collisions-see Fig. 5-and via a series of dedicated studies and analysis of operational data we were able to establish functional dependencies of the effects on such key parameters as the opposite beam intensity and emittance, on the beam's own emittance, and on beam-beam separation, machine tunes, chromaticities, etc.

The first ever two-stage beam halo collimation system using bent Si crystals was installed, tested, and introduced into operation of the Tevatron collider where it has demonstrated the predicted excellent performance [51]. Since then, bent crystal systems were developed and installed in the LHC where they demonstrated excellent performance as well. Beam diagnostics was greatly improved over the collider Run II years of 2001-2011 (Schottky detectors, ionization profile monitors, new beam position monitors, etc.) [52].

\section{ATL LAW OF DIFFUSIVE GROUND MOTION}

The stability of beam orbits in large colliders is often affected by the ATL type of the ground diffusion of accelerator tunnels [53]. The ATL law approximates the variance $\left\langle d Y^{2}(L)\right\rangle=\left\langle[d Y(z)-d Y(z+L)]^{2}\right\rangle$ of the elevation difference of the points of a tunnel as a function of the lag $L$ (distance between pairs of the measurement points) averaged over all possible time intervals $T$ as

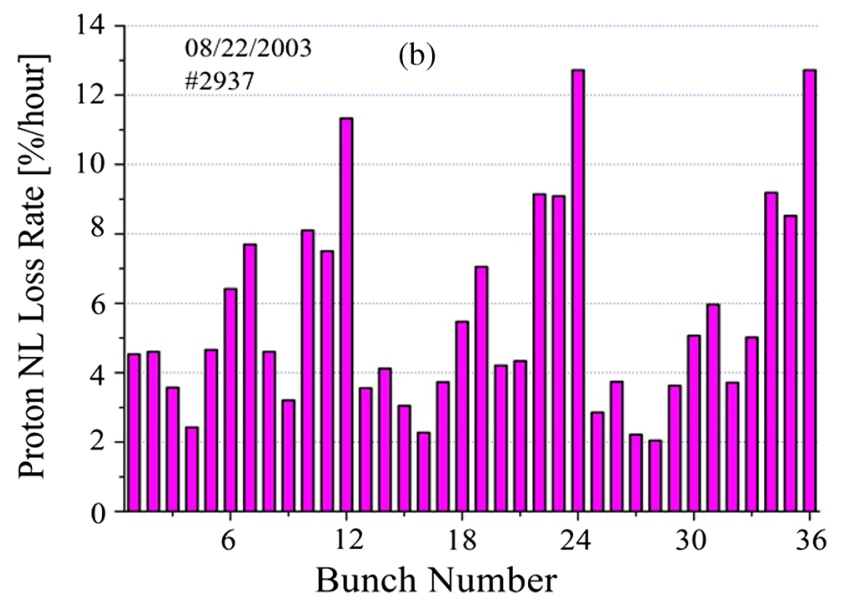

FIG. 5. (a) Left: Improvements of the Tevatron operational inefficiencies (ratios of the total bunch intensities after and before various stage) in 2001-2005; (b) right: nonluminous loss rate of proton bunch intensity at the beginning of store \#2973 (August 22, 2003)-from Ref. [49]. 


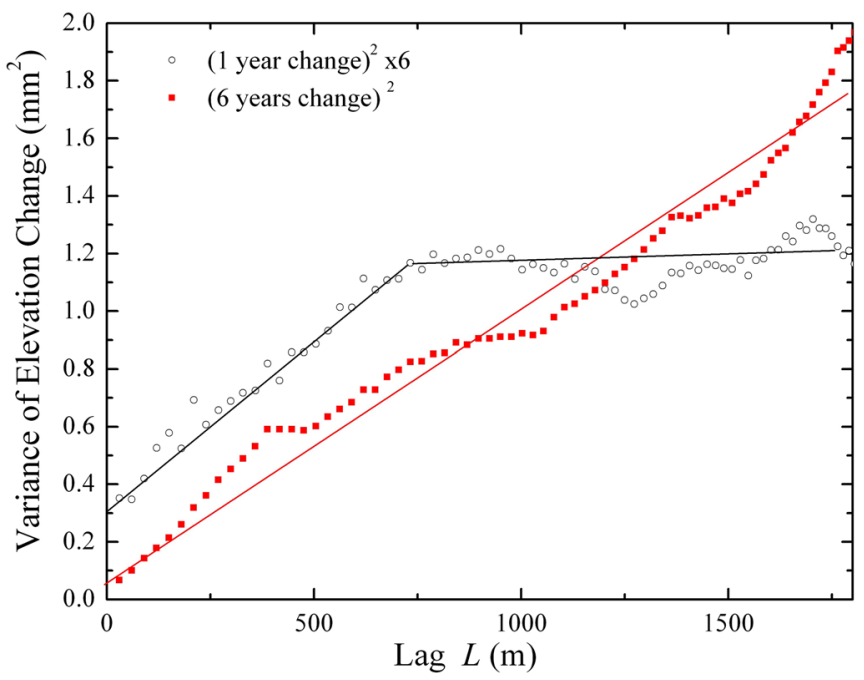

FIG. 6. Variances of the Tevatron alignment "tie rod" vertical displacements over time intervals of $1 \mathrm{yr}$ (multiplied by 6) and $6 \mathrm{yr}$ vs the distance $L$ [57]. Solid lines are according to the ATL law.

$$
\left\langle d Y^{2}\right\rangle=A T L,
$$

where $A$ is a site-dependent diffusion constant [54]. More than two decades of vibrations and ground motion studies [55] for accelerator tunnels and development of advanced instruments [56] resulted in solid experimental confirmation of the ATL law and comprehensive understanding of its practical implications for operation of all large accelerators. In particular, the Tevatron alignment "tie rod" data presented in Fig. 6 give the ground diffusion coefficient $A=$ $(4.9 \pm 0.13) \times 10^{-6} \mu \mathrm{m}^{2} / \mathrm{s} / \mathrm{m}$ [57]. Corresponding diffusive orbit deterioration due to the ground motion was clearly observed in the Tevatron, LEP, SPS, KEK-B, and TRISTAN [54].

\section{CPT THEOREM OF ACCELERATOR COMMISSIONING}

The luminosity upgrade of the Tevatron proton-antiproton collider and corresponding beam physics tests and studies resulted in a $\sim 40$-fold improvement of then the world's most powerful accelerator [58]. Accelerator physics and technology advances implemented over the course of the Tevatron operation have been comprehensively summarized in our book [59] and were of big help during beam commissioning of the LHC at CERN. Analysis of the luminosity evolution of the Tevatron which occurred as a sequence of medium- to small-scale improvements, augmented with similar observations at other accelerators, was summarized in a $C P T$ theorem for accelerators [60] that observes that in the case of the typical progress like "N\% gain per step, step after step, with regular periodicity" explains the typical exponential growth of the luminosity over time interval $T$ as $L\left(t_{0}+T\right)=L\left(t_{0}\right) \times e^{T / C}$, or

$$
C P=T,
$$

where $P=\ln \left[L\left(t_{0}+T\right) / L\left(t_{0}\right)\right]$ is the performance progress and $C$, the complexity coefficient, usually varies between $1 \mathrm{yr}$ to $3 \mathrm{yr}$ depending on the type of accelerator.

\section{VI. $\alpha \beta \gamma$ MODEL OF COST OF LARGE ACCELERATORS}

In a further analysis of various large accelerators and colliders, I turned to their costs and found out that, based on publicly available costs for 17 large accelerators of the past, the present, and those currently in the planning stage, the "total project cost (TPC)"- - sometimes cited as "the U.S. accounting" - of a collider can be broken up into three major parts corresponding to "civil construction," "accelerator components," and "site power infrastructure." The three respective cost components can be parameterized by just three parameters - the total length of the facility tunnels $L_{f}$, the center-of-mass or beam energy $E$, and the total required site power $P$ - and over almost 3 orders of magnitude of $L_{f}, 4.5$ orders of magnitude of $E$, and more than 2 orders of magnitude of $P$, the so-called " $\alpha \beta \gamma$-cost model" works with $\sim 30 \%$ accuracy [61]:

$$
\begin{aligned}
\mathrm{TPC} \approx & \alpha \times(\text { length } / 10 \mathrm{~km})^{1 / 2}+\beta \times(\text { energy } / \mathrm{TeV})^{1 / 2}+\gamma \\
& \times(\text { power } / 100 \mathrm{MW})^{1 / 2},
\end{aligned}
$$

where coefficients $\quad \alpha=2 \mathrm{~B} \$ /(10 \mathrm{~km})^{1 / 2}, \quad \gamma=2 \mathrm{~B} \$ /$ $(100 \mathrm{MW})^{1 / 2}$, while the accelerator technology-dependent coefficient $\beta$ is equal to $10 \mathrm{~B} \$ / \mathrm{TeV}^{1 / 2}$ for the superconducting rf accelerators, $8 \mathrm{~B} \$ / \mathrm{TeV}^{1 / 2}$ for normal-conducting ("warm") rf, $1 \mathrm{~B} \$ / \mathrm{TeV}^{1 / 2}$ for normal-conducting magnets, and $2 \mathrm{~B} \$ / \mathrm{TeV}^{1 / 2}$ for $\mathrm{SC}$ magnets (all numbers in 2014 U.S. dollars). This scaling law was found extremely useful for ballpark cost estimates of possible future accelerators considered in the course of the U.S. and European particle physics strategy discussions in 2013-2019.

\section{MUON COLLIDERS}

Already during the Tevatron years, I got involved in the design studies, experimental tests, and leadership of the Fermilab Muon Collider Task Force and later, in the U.S. Muon Accelerator Program. These studies were focused on the muon ionization cooling, normal conducting $\mathrm{rf}$ in strong magnetic fields, and overall machine design and established the accelerator physics feasibility of the muon collider-an alternative cost- and power-efficient energy frontier machine for the future particle physics research $[62,63]$. Now the muon colliders are under active consideration for the European particle physics strategy, with the especially attractive concept of a $14 \mathrm{TeV}$ c.m.e. $\mu+\mu-$ collider in the LHC tunnel [63]. Related activity was the development of the HTS (high temperature superconductor) 
based fast cycling magnets which could offer an economical way to build rapid cycling synchrotrons for particle physics (e.g., proton sources and muon accelerators), neutron spallation sources, and other applications [64,65]. In 2018, we have achieved the magnetic field ramping rate of $12 \mathrm{~T} / \mathrm{s}$ in a dualbore HTS magnet prototype, exceeding the previous world record rate for SC magnets by a factor of 3 [66].

\section{RESEARCH AT IOTA RING AND ELSEWHERE}

As part of the FNAL Accelerator Physics Center (APC) mission (see below), since 2009 we constructed the Fermilab Accelerator Science and Technology (FAST) facility which beside the IOTA ring comprises a $300 \mathrm{MeV} 1.3 \mathrm{GHz}$ SC rf electron linac [27]. In the fall of 2017, we achieved the world record-high superconducting of beam accelerating gradient of $31.5 \mathrm{MeV} / \mathrm{m}$ [67] — this was the first ever demonstration of an average beam accelerating gradient matching the specifications of the International Linear Collider (ILC). That accomplishment greatly boosted the confidence in the technical feasibility of the ILC - the supercollider project to push elementary particle physics beyond the LHC and which is now under serious consideration by the Japanese government. The FAST linac was also commissioned as an electron injector to IOTA ring and already supports user research [68]. Of note here is that my involvement in the linear colliders' R\&D began in the 1990s back in Russia and at DESY and, e.g., I was part of the group which for the first time demonstrated nanosecond-scale HV pulse $(\sim 5 \mathrm{kV}$, high rep rate) traveling wave injection and extraction kickers for multibunch storage rings and other accelerator applications [69], thus paving the way to many modern pulsed nanosecond HV systems based on MOSFETs, fast ionization devices (FIDs), and other fast switches and allowing one to shorten the circumference of the damping rings of the future $e+e-$ linear colliders from $10-20 \mathrm{~km}$ to about $3 \mathrm{~km}$. Also at DESY, I came out with the idea of an even faster beam-beam kicker [70] and coauthored a seminal paper on the theory of coherent synchrotron radiation [71]. In 20052007, I led the Accelerator Systems section of the U.S. LARP (LHC Accelerator Research Program) and either supervised and participated in or led the beam physics analysis, technical design, construction, or operation of such systems as high-frequency Schottky detectors, rotatable collimators, simulations of the beam-beam effects and their compensation by electron lenses, etc. All these studies helped the LHC and its upgrades as well as future proton supercolliders [72].

\section{ACCELERATOR PHYSICS CENTER}

Accelerator construction, operation, and research by necessity involve big teams of physicists, engineers, and technicians. Scientific leadership is critical for success of our activities and projects. Over more than 20 years at Fermilab, I led the beam-beam compensation project group
(1997-2001); then I was the head of the Tevatron department in 2001-2006 and was appointed the inaugural Director of the Fermilab Accelerator Physics Center (APC, 2007-2018) [73]. APC was a unique organization created in June 2007 with a mission to carry out R\&D to keep the U.S. leading high-energy physics laboratory at the forefront of accelerator science, technology, and facility operation. In support of the FNAL high-energy physics research mission, APC scientists and engineers conducted accelerator R\&D aimed at next-generation and beyond accelerator facilities; provided accelerator physics support for existing operational programs and the evolution thereof; trained accelerator scientists and engineers and established experimental programs for a broad range of accelerator R\&D that can be accessed by both Fermilab staff and by the U.S. and world's high-energy physics (HEP) community. APC was a center place for in-depth design, research, and development efforts which allowed the laboratory to make intelligent decisions on the ILC in the U.S. and on the Muon Collider, as well as to initiate projects such as the Proton Improvement Plan-II linac (PIP-II, through the Proton Driver/Project-X work), LHC-AUP (LHC Accelerator Upgrade Project, via LARP), and the IOTA/ FAST R\&D facility. The results of the APC researchers were published in 215 peer-reviewed articles and were used as an input for the discussions of the Fermilab Steering Group, of the P5 (Particle Physics Project Prioritization Panel) in 2008, during the 2014 High Energy Physics Advisory Panel (HEPAP) P5 process and the 2015 HEPAP Accelerator R\&D Subpanel meetings. Important contributions were made to the ILC CDR and TDR, to the Muon Collider design studies-see the JINST collection of reports and papers on muon accelerators [74], as well as the Project-X CDR [75] and the PIP-II CDR [76], the HighLuminosity LHC upgrade project [77], and the IOTA/FAST R\&D facility CDR [27].

Experimental beam physics R\&D was carried out at the operational accelerators at Fermilab and CERN as well as at a number of dedicated beam test facilities developed, constructed, or supported by APC for the purpose of accelerator R\&D: the Fermilab NICADD Photoinjector Laboratory with an $18 \mathrm{MeV}$ electron linac; the MuCool Test Area with a 201-MHz test cavity installed next to a $5 \mathrm{~T}$ solenoid, also used for testing the smaller $805-\mathrm{MHz}$ cavities under the impact of a $400 \mathrm{MeV}$ proton beam; the Muon Ionization Cooling Experiment (MICE) at RAL (United Kingdom) which operated with $200 \mathrm{MeV}$ muons; $325 \mathrm{MHz}$ High Intensity Neutrino Source (HINS) RFQ that accelerated protons and $\mathrm{H}$ - particles to $2.5 \mathrm{MeV} ; 1.3 \mathrm{GHz}$ SRF $300 \mathrm{MeV}$ electron injector at FAST and IOTA ring operating with up to $150 \mathrm{MeV} / \mathrm{c}$ electrons and $70 \mathrm{MeV} / \mathrm{c}$ protons.

APC also was the birthplace and host of many national and international collaborations and several educational and training programs in beam physics resulting in $27 \mathrm{Ph} . \mathrm{D}$. 
theses. In addition to the office of U.S. Particle Accelerator School (US PAS), APC hosted several programs in accelerator and beam physics education and training such as the Lee Teng Internship (jointly with Argonne National Laboratory), the Helen Edwards Internship for international students, and Joint Fermilab-University Ph.D. Program in accelerator physics. APC hosted five joint appointments with the Northern Illinois University and the Illinois Institute of Technology, as well 11 Peoples fellows, three U.S. LARP Toohig fellows, and two Imperial College London fellows. Two out of the 27 students who graduated from the Ph.D. program won the APS DPB Outstanding Doctoral Thesis Research in Beam Physics Award. In 2007-2018, APC hosted more than 100 visitors and Ph.D. or M.Sc. students, over 100 summer students in the Lee Teng and Helen Edwards programs, and some 3000 students attended semiannual US PAS sessions.

\section{NEXT STEPS: EXCITING OPPORTUNITIES}

At present, I am very interested in and participate in two research projects: the first is modeling of the space-charge compensation (SCC) with electron lenses [25] in spacecharge-dominated ultimate-intensity synchrotrons with $d Q_{\mathrm{SC}} \sim-1.0$ (jointly with E. Stern, A. Burov, and Yu. Alexahin). The simulation results show great promise of an about order of magnitude reduction in the beam losses with electron lenses-see Fig. 7 [78]. The ultimate goal of the study is to understand the requirements on and key specifications of the electron lenses for the SCC and to evaluate their effectiveness for record high beam power RCS design, as well as to make verifiable predictions for the space-charge compensation demonstration experiment in IOTA. The other project is an initial exploration of the ultimate high gradient acceleration of muons in crystals and

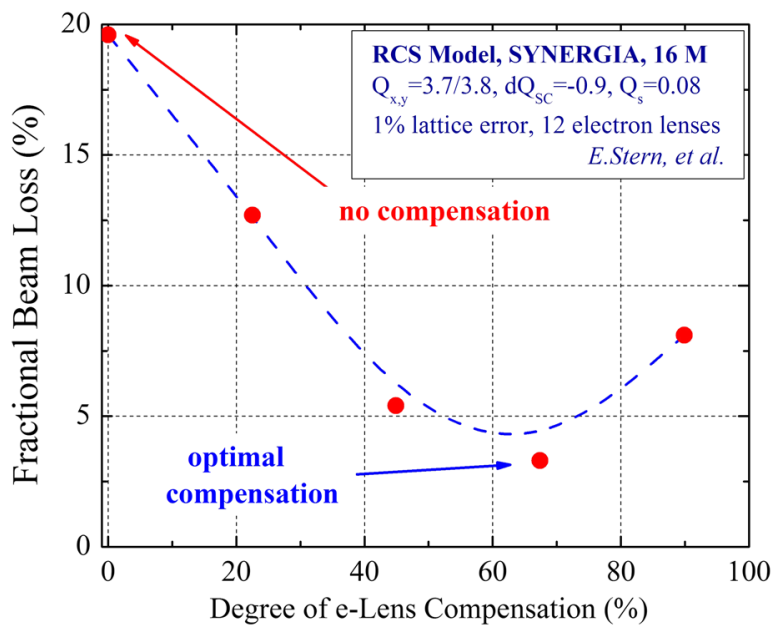

FIG. 7. Fractional loss of proton beam with $d Q_{\mathrm{SC}}=-0.9$ after 1000 turns in a model RCS with 12 electron lenses vs the degree of the SC compensation (particle-in-cell simulation data courtesy of E. Stern). nanostructures - the idea that may potentially open the path to PeV class colliders [79]. In collaboration with the pioneer of the method, Professor T. Tajima, we have organized a "Workshop on Beam Acceleration in Crystals and Nanostructures" this year at Fermilab [80].

\section{ACKNOWLEDGMENTS}

I wholeheartedly thank those who nominated me and many colleagues I had the fortune to work with over many years on the electron lenses, the Tevatron collider, and many interesting and important topics from beam-beam effects to bent crystal collimation, ground motion and orbit stabilization, head-tail instability and superfast HV pulsers, future collider designs and construction of the IOTA ring, beam commissioning of the worlds' best ILC cryomodule, and on very fast cycling HTS magnets. I would like to pay special tribute to my late collaborators D. Wildman (FNAL), G. F. Kuznetsov (BINP/FNAL), M. Tiunov (BINP), and V. Danilov (ORNL). Important contributions to the conceptual and technical development of the electron lens method were made by D. Finley, A. Valishev, G. Stancari, N. Solyak, A. Burov, E. Stern, and Yu. Alexahin (FNAL), V. Parkhomchuk and D. Shatilov (BINP), E. Tsyganov (JINR/SSCL), A. Seryi (JLab), K. Bishofberger (LANL), V. Kamerdzhiev (COSY), W. Fischer (BNL), S. Redaelli, and F. Zimmermann (CERN), and I greatly appreciate many years of fruitful collaboration with them. Fermilab is supported by U.S. Department of Energy, Office of Science, Office of High Energy Physics, under Contract No. DE-AC02-07CH11359.

[1] T. Nishikawa, Proceedings of the 9th International Conference on the High-Energy Accelerators (HEACC 1974), Stanford, CA, 1974 (SLAC, Stanford, 1974), pp. 23-31.

[2] H. Sugawara, Tetsuji Nishikawa 1926-2010, CERN-Courier 51, 31 (2011).

[3] V. Shiltsev and D. Finley, FNAL, Report No. FERMILABTM-2008, 1997.

[4] V. Shiltsev, FNAL, Report No. FERMILAB-TM-2031, 1997.

[5] V. Shiltsev, V. Danilov, D. Finley, and A. Sery, Considerations on compensation of beam-beam effects in the Tevatron with electron beams, Phys. Rev. Accel. Beams 2, 071001 (1999).

[6] V. Shiltsev, Electron Lenses for Supercolliders (Springer, New York, 2016).

[7] V. Shiltsev et al., Tevatron electron lenses: Design and operation, Phys. Rev. Accel. Beams 11, 103501 (2008).

[8] E. Tsyganov, A. Taratin, and A. Zinchenko, SSCL, Report No. 519, 1993.

[9] V. Shiltsev, Y. Alexahin, K. Bishofberger, V. Kamerdzhiev, G. Kuznetsov, and X.-L. Zhang, Experimental Demonstration of Colliding-Beam-Lifetime Improvement by Electron Lenses, Phys. Rev. Lett. 99, 244801 (2007). 
[10] X. L. Zhang, K. Bishofberger, V. Kamerdzhiev, V. Lebedev, V. Shiltsev, R. Thurman-Keup, and A. Tollestrup, Generation and diagnostics of uncaptured beam in the Fermilab Tevatron and its control by electron lenses, Phys. Rev. Accel. Beams 11, 051002 (2008).

[11] V. Shitsev, Y. Alexahin, K. Bishofberger, V. Kamerdzhiev, V. Parkhomchuk, V. Reva, N. Solyak, D. Wildman, X.-L. Zhang, and F. Zimmermann, Experimental studies of compensation of beam-beam effects with Tevatron electron lenses, New J. Phys. 10, 043042 (2008).

[12] G. Stancari, A. Valishev, G. Annala, G. Kuznetsov, V. Shiltsev, D. A. Still, and L. G. Vorobiev, Collimation with Hollow Electron Beams, Phys. Rev. Lett. 107, 084802 (2011).

[13] V. Shiltsev and V. Shiltsev, in Proceedings of the LHCLUMI-06 Workshop, Valencia, Spain, 2006 (CERN, Geneva, Switzerland, 2007) (Yellow Report No. CERN2007-002).

[14] G. Stancari, A. Valishev, G. Annala, G. Kuznetsov, V. Shiltsev, D. A. Still, and L. G. Vorobiev, Collimation with Hollow Electron Beams, Phys. Rev. Lett. 107, 084802 (2011).

[15] W. Fischer et al., Operational Head-on Beam-Beam Compensation with Electron Lenses in the Relativistic Heavy Ion Collider, Phys. Rev. Lett. 115, 264801 (2015).

[16] Conceptional Design Report No. CERN-ACC-2014-0248, 2014.

[17] D. Perini et al., in Proceedings of the 10th International Particle Accelerator Conference, Melbourne, Australia (IPAC'19) (JACoW, Geneva, 2019), MOPTS099.

[18] A. Valishev and G. Stancari, arXiv:1312.5006.

[19] S. Fartoukh, A. Valishev, Y. Papaphilippou, and D. Shatilov, Compensation of the long-range beam-beam interactions as a path towards new configurations for the high luminosity LHC, Phys. Rev. Accel. Beams 18, 121001 (2015).

[20] S. Sadovich et al., in Proceedings of the 10th International Particle Accelerator Conference, Melbourne, Australia (IPAC'19) (JACoW, Geneva, 2019), WEPTS048.

[21] V. Danilov and V. Shiltsev, Fermilab Report No. FN-671, 1998.

[22] S. Nagaitsev et al., in Proceedings of the 3rd International Particle Accelerator Conference, New Orleans, LA, 2012 (IEEE, Piscataway, NJ, 2012), MOYCP01.

[23] G. Stancari et al., in Proceedings of the 6th International Particle Accelerator Conference, Newport News, VA (IPAC'15) (JACoW, Geneva, 2015), MOBC3.

[24] V. Shiltsev, Y. Alexahin, A. Burov, and A. Valishev, Landau Damping of Beam Instabilities by Electron Lenses, Phys. Rev. Lett. 119, 134802 (2017).

[25] A. Burov, W. Foster, and V. Shiltsev, Fermilab Report No. FNAL-TM-2125, 2000.

[26] E. Stern et al., in Proceedings of the 9th International Particle Accelerator Conference, Vancouver (IPAC'18) (JACoW, Geneva, 2018), THPAF075.

[27] S. Antipov et al., IOTA (Integrable Optics Test Accelerator): Facility and experimental beam physics program, J. Instrum. 12, T03002 (2017).

[28] N. Kuklev et al., in Proceedings of the 10th International Particle Accelerator Conference, Melbourne, Australia (IPAC'19) (JACoW, Geneva, 2019), MOPGW113.
[29] I. Lobach et al., in Proceedings of the 10th International Particle Accelerator Conference, Melbourne, Australia (IPAC'19) (JACoW, Geneva, 2019), MOPRB088.

[30] L. Gupta et al., in Proceedings of the 10th International Particle Accelerator Conference, Melbourne, Australia (IPAC'19) (JACoW, Geneva, 2019), MOPGW107.

[31] S. Webb et al., in Proceedings of the 10th International Particle Accelerator Conference, Melbourne, Australia (IPAC'19) (JACoW, Geneva, 2019), MOPGW127.

[32] S. Nagaitsev et al., in Proceedings of the 10th International Particle Accelerator Conference, Melbourne, Australia (IPAC'19) (JACoW, Geneva, 2019), MOPRB089.

[33] A. Romanov et al., in Proceedings of the 10th International Particle Accelerator Conference, Melbourne, Australia (IPAC'19) (JACoW, Geneva, 2019), MOPTS115.

[34] J. Edelen et al., in Proceedings of the 10th International Particle Accelerator Conference, Melbourne, Australia (IPAC'19) (JACoW, Geneva, 2019), WEPTS068.

[35] C. Hall et al., in Proceedings of the 10th International Particle Accelerator Conference, Melbourne, Australia (IPAC'19) (JACoW, Geneva, 2019), WEPTS070.

[36] C. Hall et al., in Proceedings of the 10th International Particle Accelerator Conference, Melbourne, Australia (IPAC'19) (JACoW, Geneva, 2019), WEPTS074.

[37] K. Hwang et al., in Proceedings of the 10th International Particle Accelerator Conference, Melbourne, Australia (IPAC'19) (JACoW, Geneva, 2019), WEPTS078.

[38] C. S. Park et al., in Proceedings of the 10th International Particle Accelerator Conference, Melbourne, Australia (IPAC'19) (JACoW, Geneva, 2019), WEPTS073.

[39] C. Hall et al., in Proceedings of the 10th International Particle Accelerator Conference, Melbourne, Australia (IPAC'19) (JACoW, Geneva, 2019), MOPGW109.

[40] A. Edelen et al., in Proceedings of the 10th International Particle Accelerator Conference, Melbourne, Australia (IPAC'19) (JACoW, Geneva, 2019), WEPGW100.

[41] N. Kuklev et al., in Proceedings of the 10th International Particle Accelerator Conference, Melbourne, Australia (IPAC'19) (JACoW, Geneva, 2019), WEPGW163.

[42] T. Zolkin, in Proceedings of the 10th International Particle Accelerator Conference, Melbourne, Australia (IPAC'19) (JACoW, Geneva, 2019), FRXPLS1.

[43] T. Zolkin et al., in Proceedings of the 10th International Particle Accelerator Conference, Melbourne, Australia (IPAC'19) (JACoW, Geneva, 2019), MOPGW108.

[44] N. Kuklev, in Proceedings of the 10th International Particle Accelerator Conference, Melbourne, Australia (IPAC'19) (JACoW, Geneva, 2019), THPRB106.

[45] A. Halavanau et al., in Proceedings of the 10th International Particle Accelerator Conference, Melbourne, Australia (IPAC'19) (JACoW, Geneva, 2019), TUPRB089.

[46] V. Shiltsev, in Proceedings of the 9th European Particle Accelerator Conference, Lucerne, 2004 (EPS-AG, Lucerne, 2004), THZCH01; also Report No. Fermilab-CONF-04123, 2004.

[47] V. Shiltsev, in Proceedings of the 11th European Particle Accelerator Conference, Genoa, 2008 (EPS-AG, Genoa, Italy, 2008), TUXG02. 
[48] V. Shiltsev, Accelerator Science and Technology Breakthroughs, Achievements and Lessons from Tevatron: John Adams Lecture 2010 (CERN, Geneva, 2011), ISBN: 9290-833696.

[49] V. Shiltsev, Y. Alexahin, V. Lebedev, P. Lebrun, R. Moore, T. Sen, A. Tollestrup, A. Valishev, and X. Zhang, Beambeam effects in the Tevatron, Phys. Rev. Accel. Beams 8, 101001 (2005).

[50] N. Mokhov et al., Tevatron beam halo collimation system: Design, operational experience and new methods, J. Instrum. 6, T08005 (2011).

[51] N. Mokhov et al., Crystal collimation studies at the Tevatron (T-980), Mod. Phys. Lett. A 25, 98 (2010).

[52] R. Moore, A. Jansson, and V. Shiltsev, Beam instrumentation for the Tevatron collider, J. Instrum. 4, P12018 (2009).

[53] V. Parkhomchuk, V. Shiltsev, and G. Stupakov, Slow ground motion and operation of large colliders, Part. Accel. 46, 241 (1994).

[54] V. Shiltsev, Review of observations of ground diffusion in space and in time and fractal model of ground motion, Phys. Rev. Accel. Beams 13, 094801 (2010).

[55] B. Baklakov, T. Bolshakov, A. Chupyra, A. Erokhin, P. Lebedev, V. Parkhomchuk, Sh. Singatulin, J. Lach, and V. Shiltsev, Ground vibration measurements for Fermilab future collider projects, Phys. Rev. Accel. Beams 1, 031001 (1998).

[56] J. Volk et al., Hydrostatic level sensors as high precision ground motion instrumentation for Tevatron and other energy frontier accelerators, J. Instrum. 7, P01004 (2012).

[57] V. Shiltsev, Observations of Random Walk of the Ground in Space and Time, Phys. Rev. Lett. 104, 238501 (2010).

[58] S. Holmes and V. Shiltsev, The legacy of the tevatron in the area of accelerator science, Annu. Rev. Nucl. Part. Sci. 63, 435 (2013).

[59] Accelerator Physics at the Tevatron Collider, edited by V. Lebedev and V. Shiltsev (Springer, New York, 2014).

[60] V. Shiltsev, On performance of high energy particle colliders and other complex scientific systems, Mod. Phys. Lett. A 26, 761 (2011).

[61] V. Shiltsev, A phenomenological cost model for high energy particle accelerators, J. Instrum. 9, T07002 (2014).

[62] V. Shiltsev, When will we know a muon collider is feasible? status and directions of muon accelerator R\&D, Mod. Phys. Lett. A 25, 567 (2010).

[63] D. Neuffer and V. Shiltsev, On the feasibility of a pulsed 14 $\mathrm{TeV}$ c.m.e. muon collider in the LHC tunnel, J. Instrum. 13, T10003 (2018).
[64] H. Piekarz, S. Hays, J. Blowers, and V. Shiltsev, A measurement of HTS cable power loss in a sweeping magnetic field, IEEE Trans. Appl. Supercond. 22, 5800105 (2012).

[65] H. Piekarz, J. Blowers, S. Hays, and V. Shiltsev, Design, construction, and test arrangement of a fast-cycling HTS accelerator magnet, IEEE Trans. Appl. Supercond. 24, 4001404 (2014).

[66] H. Piekarz et al., Report No. FNAL-PUB-19-096, 2019.

[67] D. Broemmelsiek et al., Record high-gradient SRF beam acceleration at Fermilab, New J. Phys. 20, 113018 (2018).

[68] A. Halavanau, N. Eddy, D. Edstrom, E. Harms, A. Lunin, P. Piot, A. Romanov, J. Ruan, N. Solyak, and V. Shiltsev, Analysis and measurement of the transfer matrix of a 9-cell, 1.3-GHz superconducting cavity, Phys. Rev. Accel. Beams 20, 040102 (2017).

[69] B. I. Grishanov, F. V. Podgorny, J Rümmler, and V. D. Shiltsev, Very fast kicker with high repetition rate for accelerator applications, Nucl. Instrum. Methods Phys. Res., Sect. A 396, 28 (1997).

[70] V.D. Shiltsev, Beam-beam kicker for superfast bunch handling, Nucl. Instrum. Methods Phys. Res., Sect. A 374, 137 (1996).

[71] Ya. Derbenev, J. Rossbach, E. Saldin, and V. Shiltsev, DESY Report No. TESLA-FEL 95-05, 1995.

[72] V. Shiltsev, Accelerator physics and technology challenges of very high energy hadron colliders, Int. J. Mod. Phys. A 30, 1544001 (2015).

[73] V. Shiltsev, Report No. Fermilab-FN-1059, 2018.

[74] JINST Special Issue Muon Accelerators for Particle Physics, https://iopscience.iop.org/journal/1748-0221/page/ extraproc46.

[75] Project-X CDR, Report No. FERMILAB-TM-2557, 2013.

[76] V. Lebedev et al., The PIP-II Conceptual Design Report, v.0.02, Fermilab document PIP-II-doc-113, 2018, http:// pxie.fnal.gov/PIP-II_CDR/default.htm.

[77] G. Apollinary et al., arXiv:1705.08830.

[78] E. Stern, Yu. Alexahin, A. Burov, and V. Shiltsev, Fermilab internal beams-document-6790, 2019.

[79] V. Shiltsev, High-energy particle colliders: The past 20 years, the next 20 years, and the distant future, Phys. Usp. 55, 965 (2012).

[80] Proceedings of the Workshop on Beam Acceleration in Crystals and Nanostructures, Fermilab, 2019 (to be published); https://indico.fnal.gov/event/19478/. 